\title{
AC 2012-3923: MEASUREMENT OF OP-AMP PARAMETERS USING VEC- TOR SIGNAL ANALYZERS IN UNDERGRADUATE LINEAR CIRCUITS LABORATORY
}

\author{
Dr. Tooran Emami, U.S. Coast Guard Academy
}

Tooran Emami received her M.S. and Ph.D. degrees in electrical engineering from Wichita State University (WSU) in 2006 and 2009, respectively. She is an Assistant Professor in Department of Engineering at the U.S. Coast Guard Academy. Her research interests are in Proportional Integral Derivative (PID) controllers, robust control, time delay, and compensator design for continuous-time, and discrete-time systems.

\section{Prof. Richard J. Hartnett, U.S. Coast Guard Academy}

Richard J. Hartnett is a professor of electrical engineering at the U.S. Coast Guard Academy in New London, Conn. He received his B.S.E.E. degree from the U.S. Coast Guard Academy, the M.S.E.E. degree from Purdue University, and his Ph.D. in E.E. from the University of Rhode Island. His research interests include efficient digital filtering methods, improved receiver signal processing techniques for electronic navigation systems, and autonomous vehicle design. 


\title{
Measurement of Op-Amp Parameters \\ Using Vector Signal Analyzers in an \\ Undergraduate Linear Circuits \\ Laboratory
}

\begin{abstract}
Measurements of op-amp circuit parameters such as open or closed-loop frequency response, or output impedance as a function of frequency, over a reasonably broad range of frequencies, can be tedious and very challenging for undergraduate students to accomplish using signal generators and oscilloscopes. In fact, measurements of total harmonic distortion (measure of op-amp linearity), with and without resistive load, are almost impossible to make in the time domain with conventional oscilloscopes. Of greater concern is that the time-domain methods for measuring frequency domain characteristics of an op-amp do not present a "real-time" visualization for students. This problem can even mask important operational limitations such as op-amp slewrate nonlinearities for higher frequency sinusoidal inputs.

This paper presents some of the successful measurement methodologies that our students use at the U.S. Coast Guard Academy in a junior-level Linear Circuits laboratory. As part of this lab, students use the Agilent 35670 Dynamic Signal Analyzer (DSA) to measure some of the specifications of an inverting amplifier op-amp ( $\mu$ A741) circuit. They use averaging on the measurement data to minimize the impact of noise in the measurements of the $\mu$ A741circuit. Here we present typical measurement results, along with informal student feedback that suggests to us that the "real-time" nature of a DSA frequency domain presentation (that looks almost exactly like Matlab ${ }^{\mathrm{TM}}$ and MultiSim ${ }^{\mathrm{TM}}$ predictions) really does reinforce student learning.

The lab procedure consists of four steps: (1) Students set the DSA to measure the open-loop frequency response of the op-amp over two different frequency ranges. (2) Students then measure the closed-loop frequency response of an inverting amplifier for two different gain settings. (3) Students measure the output impedance of an inverting amplifier circuit as a function of frequency. (4) Finally, they compare the Total Harmonic Distortion (THD) at the output and differential input, for unloaded and resistive load conditions. Frequency response measurements are compared with theoretical expectations from Matlab ${ }^{\mathrm{TM}}$ and with MultiSim ${ }^{\mathrm{TM}}$ $\mathrm{AC}$ analysis simulations, thereby solidifying the frequency domain presentation of real-world opamp characteristics.
\end{abstract}

\section{Introduction}

Measuring Operational Amplifier (op-amp) parameters can represent one of the most challenging laboratories for undergraduate students. For the most part, many of these challenges stem from the fact that classical time-domain methods for measuring op-amp "frequency domain 
parameters" can be cumbersome, and typically do not present a "real-time" visualization for the student. Parameters such as gain-bandwidth product, open and closed loop frequency responses, and Total Harmonic Distortion (THD) at the op-amp output and differential input (under loaded and unloaded conditions) are inherently easier to interpret, compute, and visualize in the frequency domain.

Some of the early ideas for considering the effects of design with "real-world" op-amps originated with Peterson, Hartnett, and Gross at the U.S. Coast Guard Academy ${ }^{1}$, however the authors focused primarily on the theoretical analysis and did not discuss measurement methodologies. Classic references such as Schaumann and Van Valkenburg ${ }^{2}$ and Van Valkenberg ${ }^{3}$ also discuss the idea of "one-pole roll-off" and "two-pole roll-off" real-world opamp models, but present no measurements or methodologies.

In this paper we introduce successful measurement of op-amp parameters that our undergraduate students achieve at the U.S. Coast Guard Academy in a junior-level Linear Circuits laboratory. Students use Agilent 35670A Dynamic Signal Analyzers (DSA's) to measure the open-loop and closed-loop frequency responses, output resistance, and THD (at output and inverting input) of an inverting amplifier circuit using a $\mu$ A741 op-amp. From their DSA measurements of frequency response, THD, and output resistance, students learn that even a low-cost $\mu$ A741 opamp can behave as an "ideal amplifier" in a circuit, if one operates the amplifier within the gainbandwidth product, slew rate, and output current limitations of the selected op-amp. All frequency response measurements are then verified with theoretical expectations from Matlab ${ }^{\mathrm{TM}}$ and MultiSim ${ }^{\mathrm{TM}} \mathrm{AC}$ analysis simulations.

\section{Laboratory Procedures}

The lab procedure consists of four steps: (1) Students set the DSA to measure the open-loop frequency response of the op-amp over two different frequency ranges. (2) Students then measure the closed-loop frequency response of an inverting amplifier for two different gain settings. (3) Students measure the output impedance of an inverting amplifier circuit as a function of frequency. (4) Finally, students measure the total harmonic distortion at $V_{\text {- and }} \mathrm{V}_{\text {out }}$ for a (clean) sinusoidal input, for loaded and unloaded inverting amplifier configurations.

In the first step, students are instructed to build the circuit shown in Figure 1 using a $\mu$ A741 opamp. After verifying proper operation, they begin measuring the $\mu \mathrm{A} 741$ op-amp open loop frequency response; that is the transfer function of the op-amp itself from the inverting terminal $\mathrm{V}_{\text {- to }} \mathrm{V}_{\text {out }}$. The general idea in this measurement is to provide the circuit input with a periodic chirp waveform, from the "source output" of the DSA, over the frequency range of interest. Connecting Channel\#1 of the DSA to V. and Channel\#2 of the DSA to $\mathrm{V}_{\text {out }}$ of the circuit, coupled with selecting a "frequency response" measurement, yields a measurement of open-loop magnitude response for the student. 


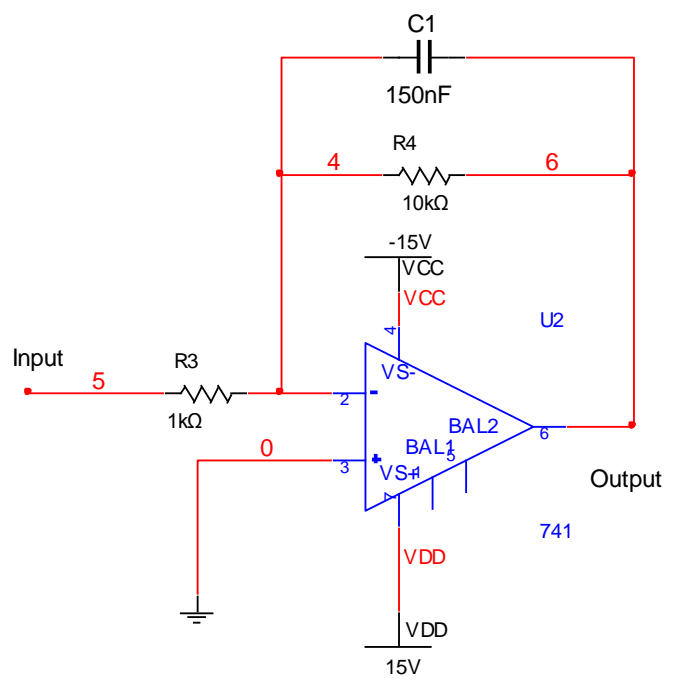

Figure 1. Op-amp circuit used to measure open loop frequency response of $\mu \mathrm{A} 741 \mathrm{op}-\mathrm{amp}$.

One might ask why it would matter what circuit our students use if we are interested in an openloop frequency response measurement (i.e. from $\mathrm{V}_{\text {- }}$ to $\mathrm{V}_{\text {out }}$ ). The short answer is that an op-amp circuit is considerably more stable with negative feedback, so it is often much easier to measure open-loop characteristics operating within a closed loop system. The longer answer regarding why this particular circuit is used is perhaps more subtle. One might initially be tempted to have students simply use an inverting amplifier circuit such as one shown in Figure 2. In this circuit, assuming a periodic chirp input (which is spectrally flat), and taking a closer look at the voltage $\mathrm{V}$. (the op-amp inverting input) as a function of frequency, we realize that the amplitudes of the low frequency components are extremely small compared to the higher frequency components. As a result, the A/D range for Channel \#1 on the DSA would be dominated by high frequency components, and low frequency (low amplitude) measurements of $\mathrm{V}$ - (which form the denominator of the open-loop frequency response) would render open-loop frequency response measurements extremely challenging for students to make.

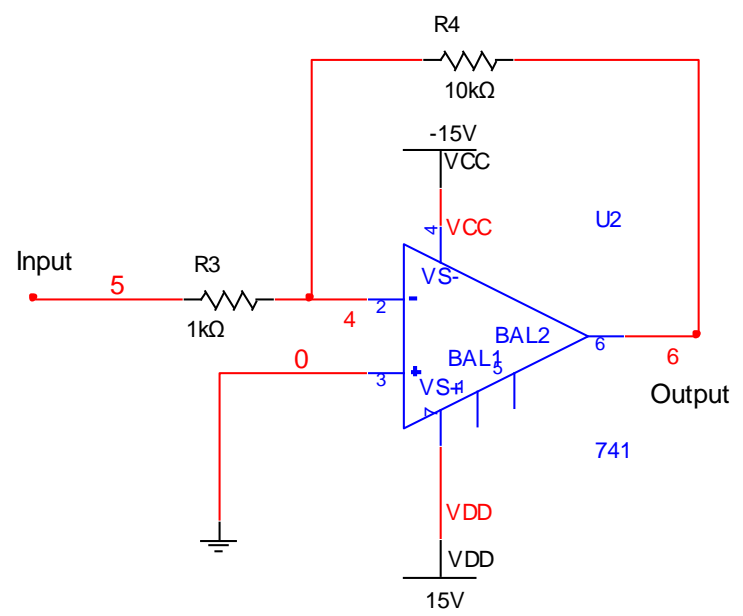

Figure 2. Typical inverting amplifier configuration (showing a linear gain of -10). 
In having our students use the low pass amplifier configuration from Figure 1, the node voltage at $\mathrm{V}$ - is considerably more level as a function of frequency, which contributes to higher quality measurements of open-loop frequency response.

The initial DSA settings for the open-loop circuit are shown in Table 1. Note, we use digital averaging (available as a choice on the Agilent 35670) to filter measurement noise. For this

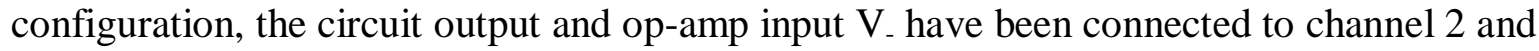
channel 1 of the DSA, respectively.

Table 1

Initial DSA Setting for Open-loop Frequency Response Measurement

\begin{tabular}{ll}
\hline Parameter & Setting \\
\hline Source & On/Periodic Chirp \\
Input & $600 \mathrm{mVrms}$ \\
Measure & Freq Response $(\mathrm{Ch} 2 / \mathrm{Ch} 1)$ \\
Freq & Start/0\&Span/400 Hz \\
Trace coordinate & dB/Mag \& x-axis/log \\
Average & On/100 \\
Auto Scale & On/off \\
\hline
\end{tabular}

There are two measurements for the open loop frequency response, spanning the frequency ranges of 1 to $400 \mathrm{~Hz}$ and 128 to $51200 \mathrm{~Hz}$. The open-loop measurement magnitude response of the 741 op-amp in the range of 1 to $400 \mathrm{~Hz}$ is shown in Figure 3. As measured, the open-loop frequency response has a DC gain of approximately $109 \mathrm{~dB}$ (280000), and a break frequency of about $4 \mathrm{~Hz}$. From the measurement data on the linear portion of the magnitude response curve from Figure 3, the gain bandwidth product can be calculated. Here the gain-bandwidth is approximately $1.1 \mathrm{MHz}$.

$$
\begin{aligned}
G B P & =100\left(10^{80.87 / 20}\right) \\
& =1.1 \mathrm{MHz}
\end{aligned}
$$

These results can be compared with the theoretical specifications of a real $\mu \mathrm{A} 741$ op-amp which predict an open loop DC gain of about $106 \mathrm{~dB}$ (200000), a break frequency of about $5 \mathrm{~Hz}$, and a gain bandwidth product of approximately $1 \mathrm{MHz}$ (shown in Figure 4). 


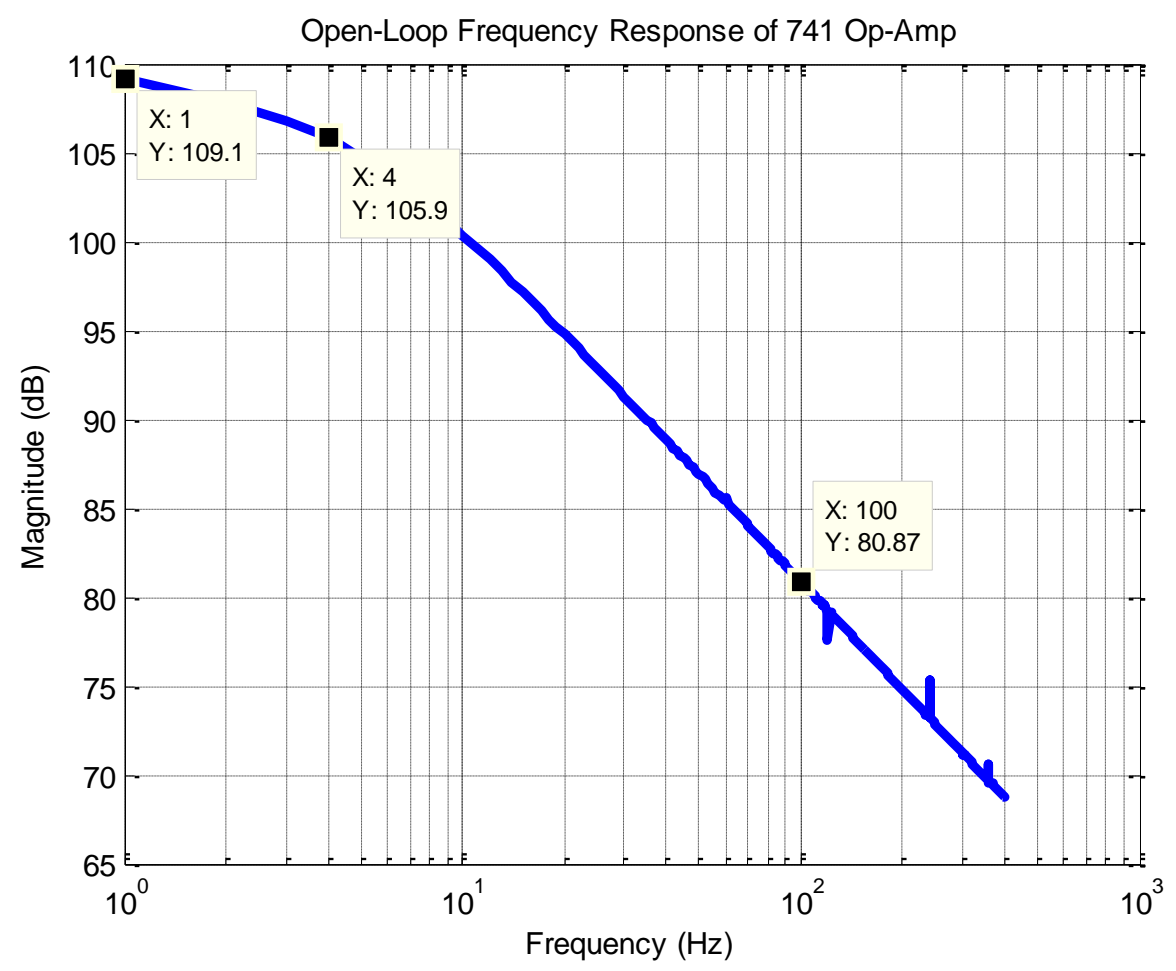

Figure 3. Open-loop magnitude response (measured) for a $\mu$ A741 op-amp.

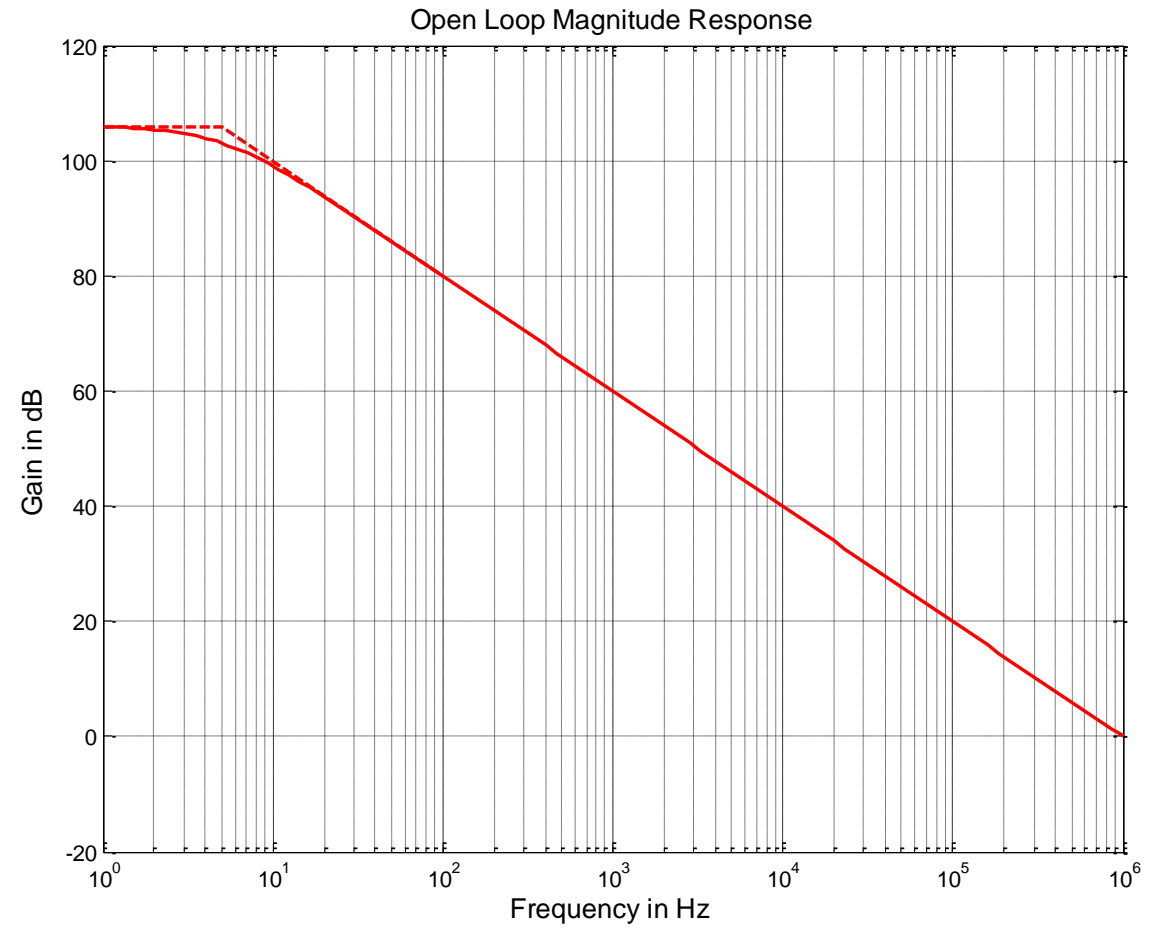

Figure 4. Open-loop magnitude response (theoretical) for a $\mu$ A741 op-amp.

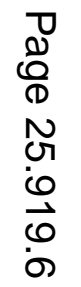


In the second step of the lab, students measure the closed-loop magnitude response for an inverting amplifier (Figure 2) for two different gains (-10 and -100), over a frequency range of $128 \mathrm{~Hz}$ to $51.2 \mathrm{kHz}$. Settings from Table 1 are used; however the DSA periodic chirp amplitude routed to the circuit input is decreased to $10 \mathrm{mV}_{\text {rms }}$ to reduce high frequency slew rate nonlinearities. The open-loop and closed-loop magnitude response measurements for two different gains (-10 and -100) are shown in Figure 5. Theoretical open-loop and closed-loop magnitude response predictions are shown in Figure 6.

After seeing both theoretical and measured results in the frequency domain, students appreciate that inverting amplifiers using "real-world" op-amps cannot provide constant gain over an infinite range of frequencies. Furthermore, in overlaying the open-loop characteristic with the closed-loop characteristic, students understand that "real-world" op-amps circuits seem to behave like "ideal" op-amp circuits if we restrict the input signal bandwidth. Deviation from ideal op-amp behavior in an inverting amplifier circuit occurs when we attempt to exceed the gain-bandwidth limitation of the op-amp itself. At that point, the closed-loop magnitude response seems to be dominated by the open-loop characteristic of the chosen op-amp, and students readily see this as they make their DSA measurements, perform MultiSim ${ }^{\mathrm{TM}} \mathrm{AC}$ analysis simulations, and plot predicted magnitude responses from Matlab ${ }^{\mathrm{TM}}$.

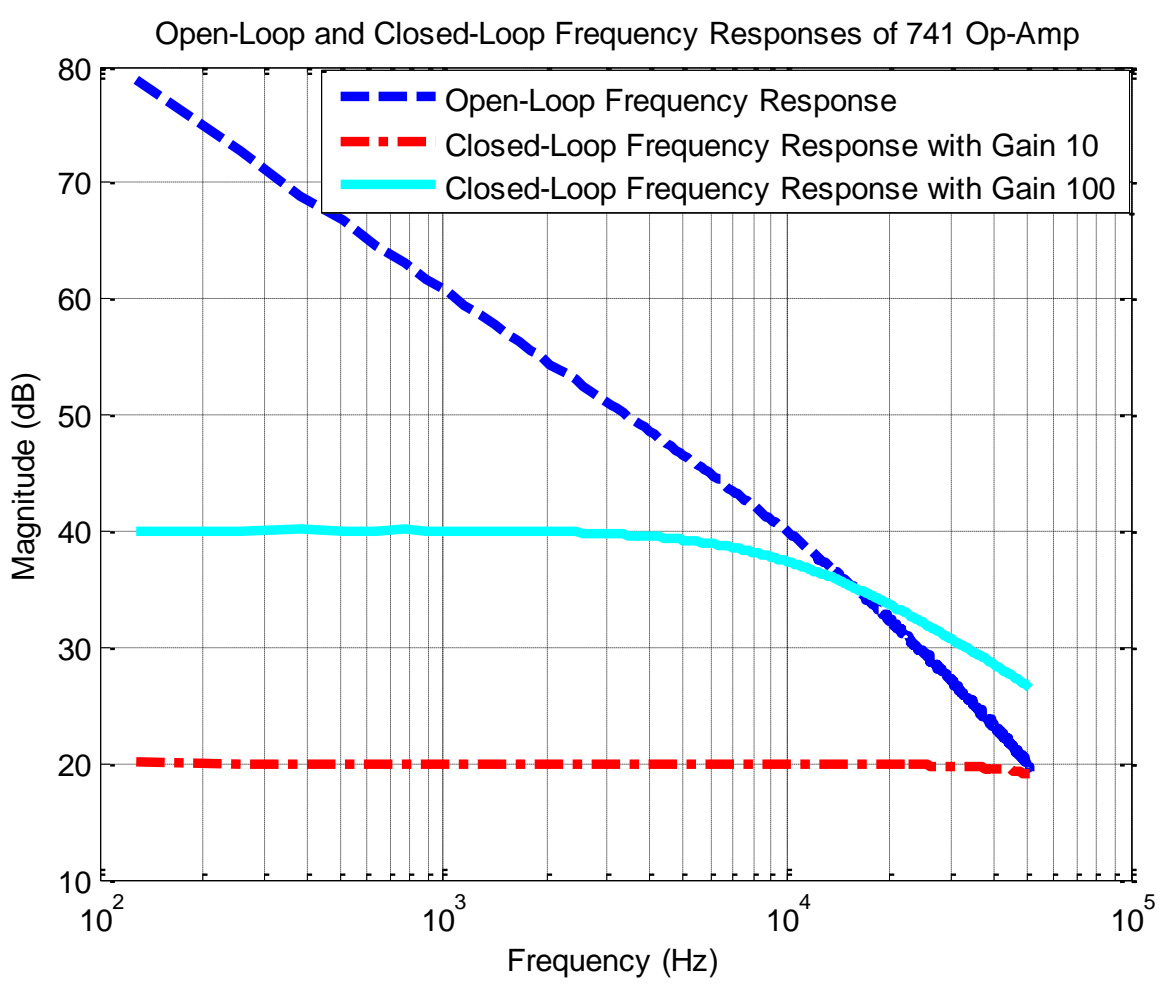

Figure 5. Open-loop and closed-loop magnitude responses (measured). 


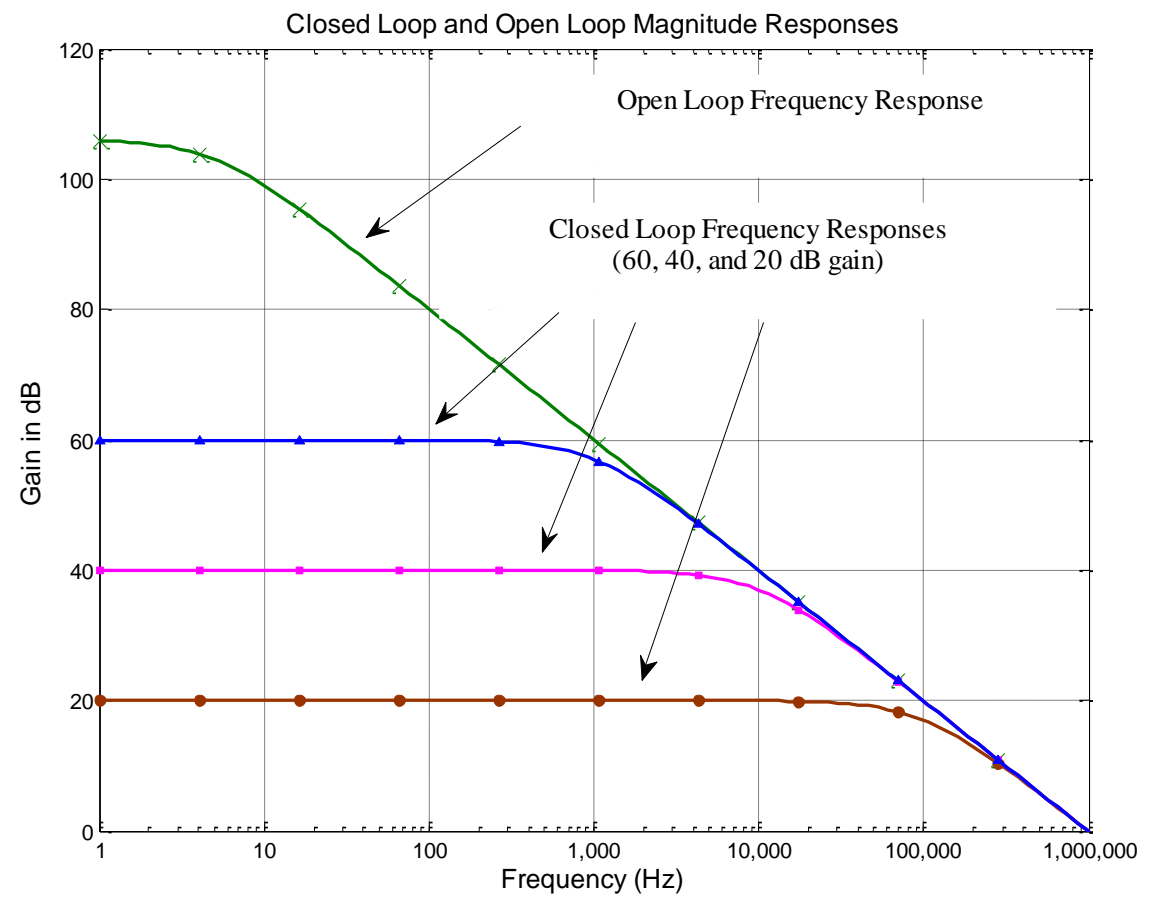

Figure 6. Open-loop and closed-loop magnitude responses

(Predicted from MatlabTM, where a $1^{\text {st }}$ order transfer function has been assumed for the op-amp).

In the third step of the lab, a $200 \Omega$ load resistance is connected at the output of the amplifier (to ground) with a gain of -100 (Figure 7). The same settings from Table 1 are used in this step, however the periodic chirp amplitude from DSA routed to the circuit input is decreased to 10 $\mathrm{mV}_{\text {rms }}$ (to minimize high frequency slew rate nonlinearities, and to keep output currents below the rated currents for a $\mu \mathrm{A} 741 \mathrm{op}-\mathrm{amp}$ ). At this point, students immediately see (in real-time) the effect of loading and unloading the amplifier circuit (Figure 8). They see that applying a load resistor causes the magnitude response curve to shift downward at higher frequencies, and they see that the curve does not shift downward very much at lower frequencies.

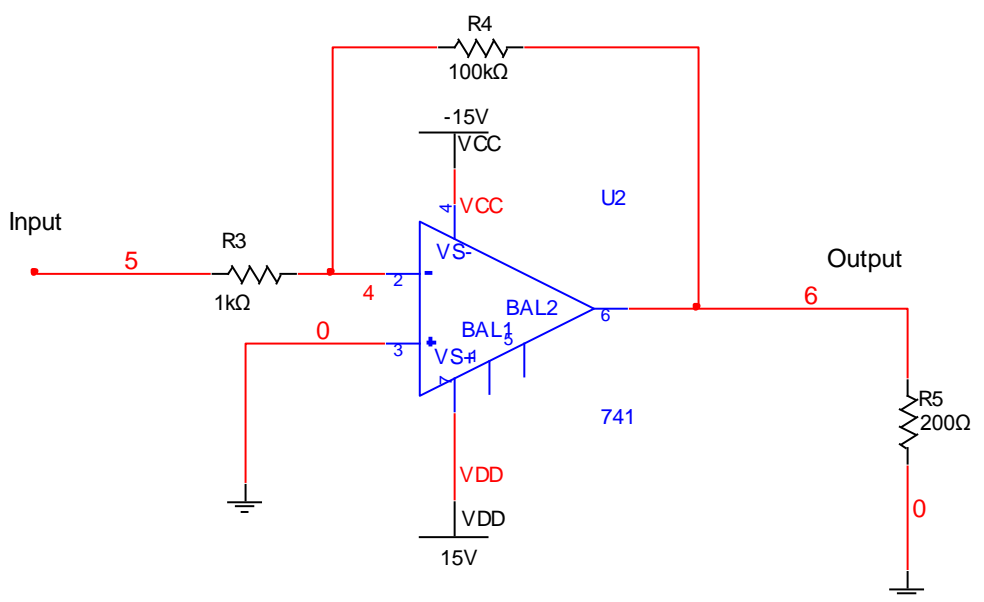

Figure 7. Inverting amplifier configuration (gain of -100) showing load resistor. 


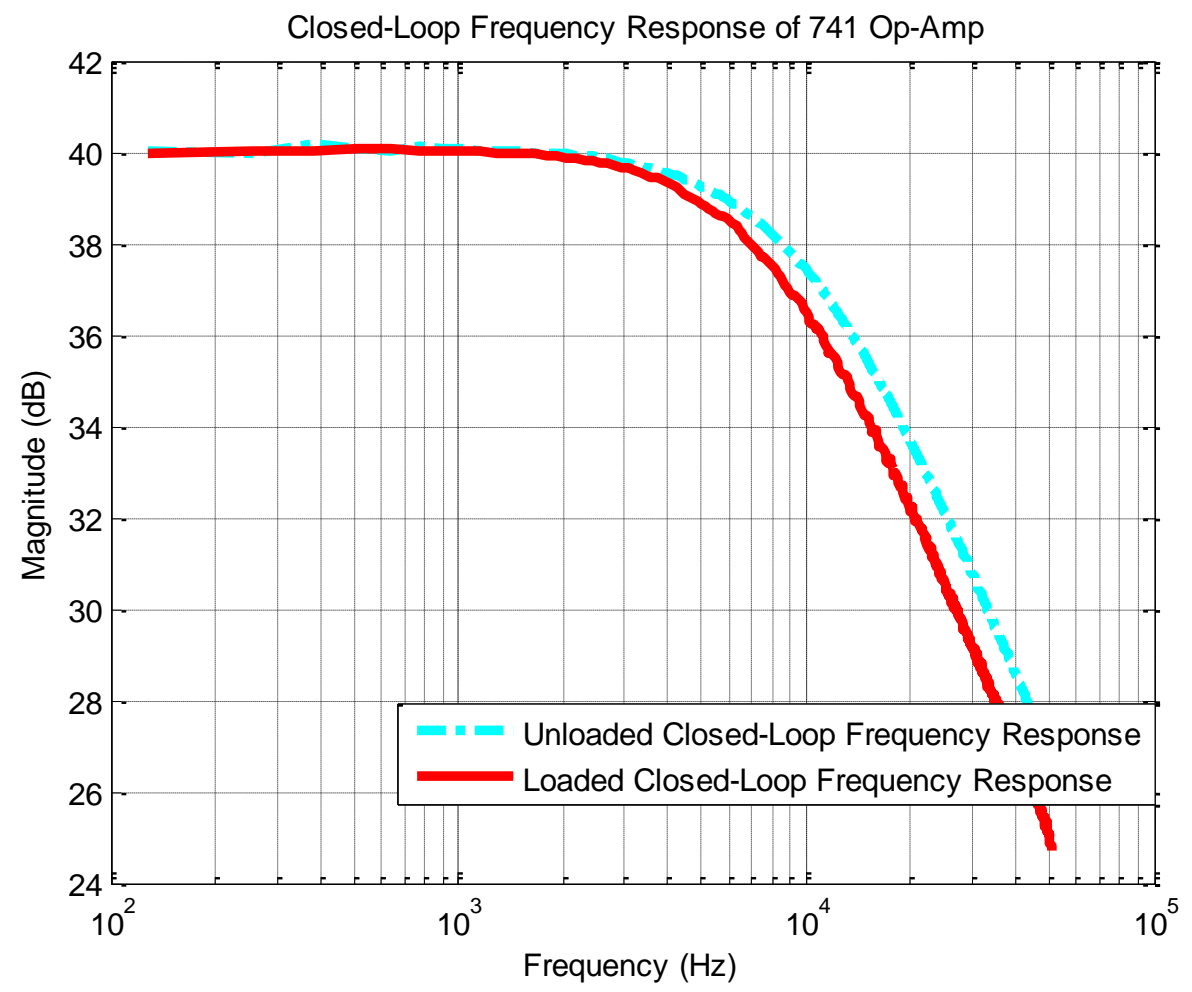

Figure 8. Measured magnitude response of the loaded and un-loaded inverting amplifier circuit with $\mu$ A741 op-amp.

The output impedance of the amplifier can be found by calculating the difference between the magnitude response of the loaded and unloaded frequency response. More specifically, examining the output side of Figure 7, we could consider this real world op-amp as an ideal opamp (with zero output impedance) in series with $R_{\text {out }}$ (Figure 9). By adding the load resistor, students use voltage division techniques to calculate Rout as a function of frequency.

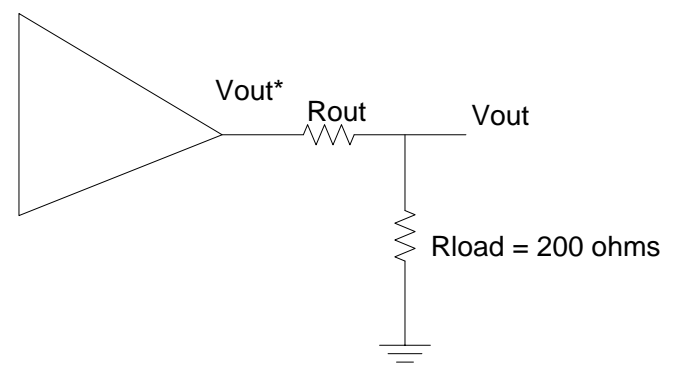

Figure 9. Simplified diagram for circuit from Figure 7,

where we treat the real world op-amp as an ideal op-amp in series with $\mathrm{R}_{\text {out }}$.

The measured amplifier output impedance is shown in Figure 10. Note that the amplifier impedance is very close to zero at low frequency (meaning that the amplifier behaves in an ideal way), and it goes up to about $43 \Omega$ above $40 \mathrm{kHz}$ (or as we approach the portion of the gain- 
bandwidth curve that is dominated by the open-loop op-amp characteristic). From this investigation, students realize again that "real-world" op-amps circuits seem to behave like "ideal" op-amp circuits if we restrict the input signal bandwidth.

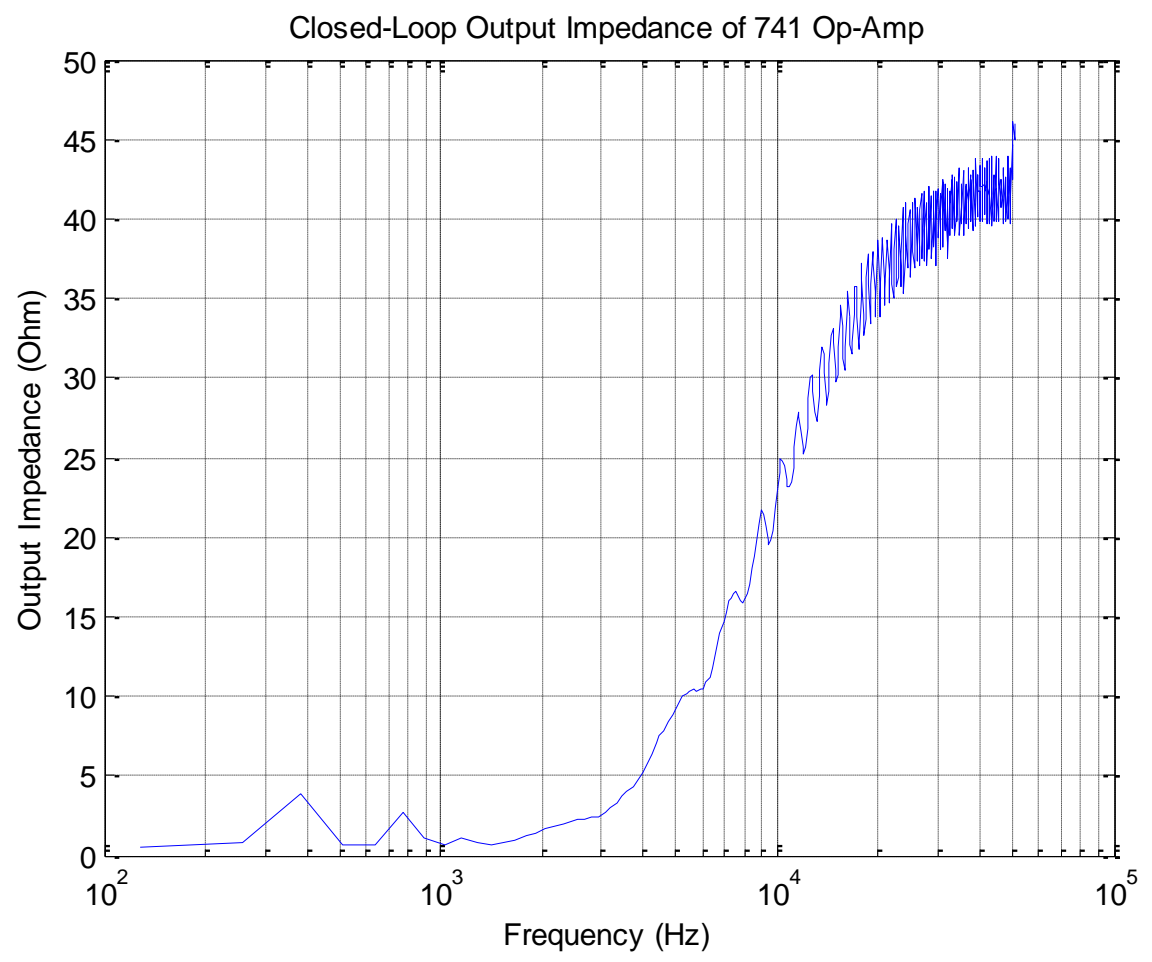

Figure 10. Output impedance as a function of frequency for inverting amplifier configuration.

In the fourth step of the lab, students measure the total harmonic distortion of a resistive loaded inverting amplifier, constructed with a 741 op-amp. The amplifier is designed for a gain of -3 . The measurements shown here are the closed-loop circuit output and differential input of total harmonic distortions for resistive loaded and unloaded conditions.

One important feature of op-amp circuits with negative feedback is that the closed loop system will often be highly linear even if the op-amp itself displays some nonlinearities. The normal method for measuring the linearity of an amplifier is to use a pure sinusoid as an input and measure the THD of the output. The effect of any non-linearity is to generate harmonics of the input frequency. Total Harmonic Distortion relates the root-squared sum (RSS) of the harmonic components to the amplitude of the fundamental, and is usually expressed either in $\mathrm{dB}$ or as a percent.

The DSA settings for this measurement are given in Table 2. Figure 11 shows the spectra of both the output (top trace) and the differential input ( $\mathrm{V}_{-}$) (bottom trace) for the unloaded inverting amplifier with gain of -3 . Note however, that the output is almost a perfect sinusoid, 
indicating the closed loop system is highly linear, while the differential input voltage is slightly more distorted.

Table 2

DSA Setting for Total Harmonic Distortion

\begin{tabular}{ll}
\hline Key Bottom & Selected \\
\hline Source & On/Fixed Sine \\
Input & 3.5 Vot $(0-$ Peak $)$ \\
Measure & Pwr Spec/Ch 2 \\
Auto Scale & On/off \\
Trace coordinator & Log/Mag \& x-axis/lin \\
Marker Function & Harmonic /THD \\
Display Format & Up/Down \\
Active Trace & A/Output \\
Active Trace & B/ Differential Input \\
Freq & Start/1024 Hz- Span/12.8 KHz \\
\hline
\end{tabular}

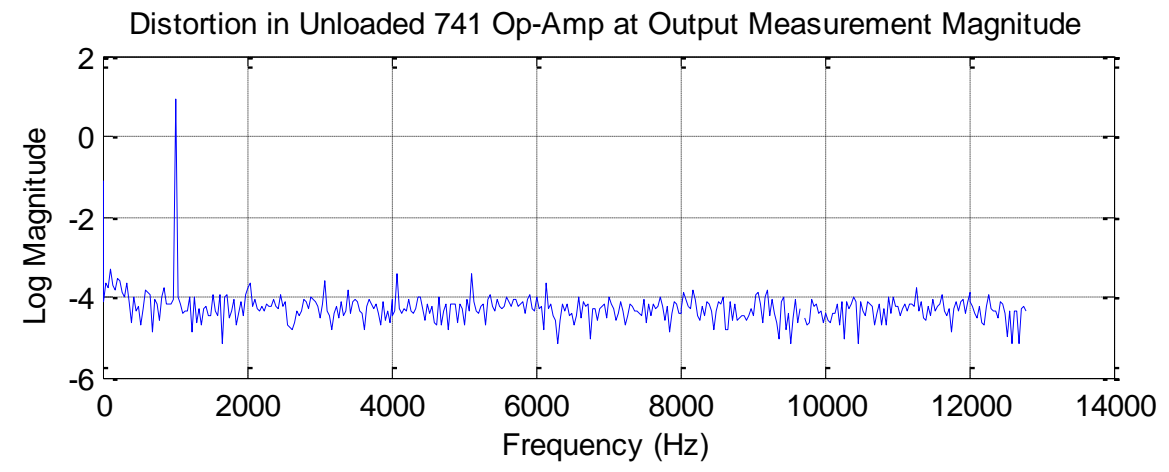

Distortion in Unloaded 741 Op-Amp at Differential Input Measurement Magnitude

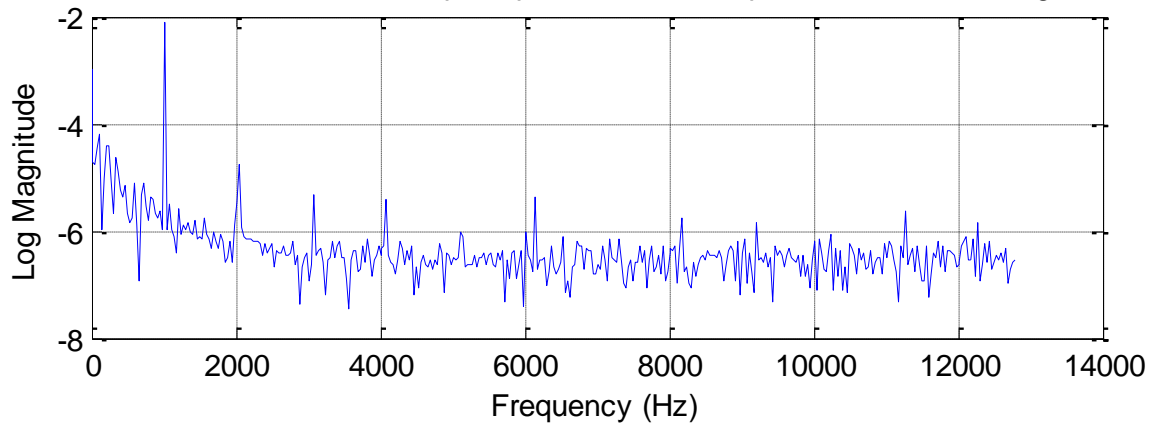

Figure 11. The output distortion and the differential input distortion of unloaded amplifier (for pure sinusoidal input).

More dramatic is to measure distortion at circuit output and differential input of the loaded amplifier (again, with gain of -3). A load resistor of $1 \mathrm{k} \Omega$ is connected at the output where the DSA settings are given in Table 2. The output distortion and the differential input distortion of 
loaded amplifier are shown in Figure 12. Note that the op-amp itself becomes much more nonlinear when connected to a resistive load. The closed loop system is still quite linear even though the open loop system is now much more non-linear than before, as evidence by the THD (approximately 6\%) at the differential input. Note the significant distortion in the differential input, while the output is relatively undistorted. From this exercise, students learn that a "realworld" op-amp can in fact exhibit nonlinearities (especially as it drives more of a load), and that negative feedback makes a circuit with an "imperfect" op-amp behave in a much more "ideal" (linear) way. Said another way, a non-linear open loop system becomes a linear closed loop system.
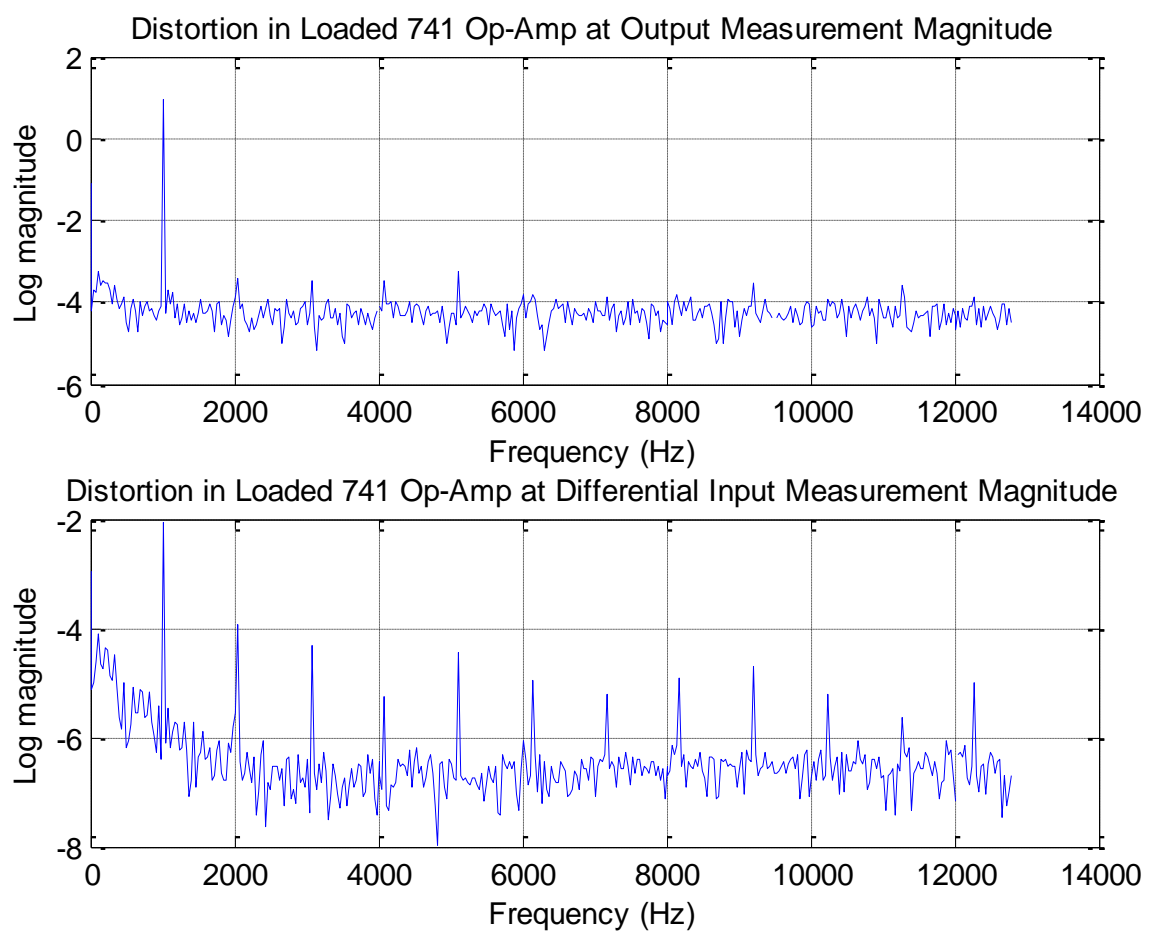

Figure 12. Output distortion and differential input distortion of loaded amplifier.

\section{Conclusion}

A successful methodology was provided to students in order to measure $\mu$ A741op-amp circuit parameters in the frequency domain. As part of this lab, students used the Agilent 35670A Dynamic Signal Analyzer (DSA) to measure the open-loop and closed-loop frequency responses, and output resistance, of an inverting amplifier circuit. They also compared the Total Harmonic Distortion (THD) at the output and inverting input, for unloaded and resistive load conditions. All measurements were relatively easy to accomplish using the Agilent 35670A, and provided students a tremendous visual tool as they completed their lab exercises. Student learning was reinforced by using Matlab ${ }^{\mathrm{TM}}$ predictions, MultiSim ${ }^{\mathrm{TM}}$ simulations, and measurements, all in the frequency domain. Informal student feedback and results from one hourly examination suggest 
to us that students learned exactly how real world op-amps differ from ideal op-amps. The most importantly, they learned that a real-world op-amp in a circuit with negative feedback can appear as if it is ideal, if one does not try to push the gain-bandwidth or slew rate capabilities of the device.

\section{References:}

1. Peterson, B., Hartnett, R., and Gross, K., Analog and Digital Filter Design, unpublished textbook, 2011.

2. Schaumann, R., and Van Valkenburg, M., Design of Analog Filters, Oxford University Press, Inc., New York, 2001.

3. Van Valkenburg, M., Analog Filter Design, Holt, Rinehart and Winston, Inc., New York, 1982. 